International Journal of Engineering \& Technology, $7(2.24)(2018) 531-535$
International Journal of Engineering \& Technology
SPC
Website: www.sciencepubco.com/index.php/IJET
Research paper

\title{
Huffman coding packet balancer based data compression techniques in Wireless Sensor Network
}

\author{
Varun Rao ${ }^{1 *}$, Sandeep Nukala ${ }^{2}$, Abirami $\mathbf{G}^{3}$, Deepa $\mathbf{R}^{4}$, Revathi Venkataraman $^{5}$ \\ 1,2,3,4,5 Computer Science \&amp; Engineering \\ SRM Institute of Science and Technology, Kattankulathur, Chennai, India \\ *Corresponding Author E-mail: raovarun1123@gmail.com
}

\begin{abstract}
In Wireless Sensor Networks, sensor devices perform sensing and communicating task over a network for data delivery from source to destination. Due to the heavy loaded information, during packet transmission, sensor node will drain off its energy frequently, thus led to packet loss. The novelty of the proposed work is mainly reducing the loss of packet and energy consumption during transmission. Thus, Huffman coding packet balancer select the best path between the intermediate nodes and are compared based on transmitting power, receiving and sensing power these measure the QOS in wireless sensor network. To satisfy the QOS of the node, compressed packet from source to destination is done by choosing the best intermediate node path. The advantages of the proposed work is minimum packet loss and minimize the end to end delay. Sparse recovery is used to reconstruct the path selection when there is high density of node.
\end{abstract}

Keywords: Sparse Recovery, Compressed Packet, Huffman Coding Packet Balancer.

\section{Introduction}

In the present scenario, wireless sensor networks are playing a vital role with their versatility and have been deployed in several applications like monitoring various life activities from health to agriculture. In many of these applications various sensors are deployed and they require the sensors to sense and collect the data. Once deployed these sensors will collect the data and transmit the data from node to node through multi-hop paths.Inwireless sensor network the sensor nodes are used to send and transmit information in form of packets from source to destination. Due to the heavy load information in packet transmission the sensor node will drain up its battery and causes limitation in energy efficiency.

Compressed sensing is a technique in which the data transfer from node to node can be made with the signal processing, finding a path to reach the destination. Transferring the data that is compressed may lead to problems like packet loss between intermediate nodes, exceeding energy consumption may lead to drain the energy which results in minimising the residual energy. Also it may lead to problems regarding the computation cost which will have chances of less security. The Lempel zivcompression technique is used to compress the data to make the data transfer easy and reduce the time taken to transfer. Sparse is the path through which the node that is residing far from the destination in the deepest will not find a path. To overcome this, the sparse recovery is additionally made so that, the node will find the shortest path with the intermediate nodes. Taking various parameters into consideration the shortest path will be taken and one node to other the data will transfer. The nodes will be shown in matrix form in which some nodes will get null value and to overcome this with some random value and to find the shortest path to reach the destination. In existing work, the compressed sensing is used to transfer the data to reduce load by reducing the packet size and then transfer the data. The source to destination data transfer is done by finding the shortest path using the grid topology [5]. The data transfer is taking maximum time due to the excess power consumption which leads to energy loss. While finding the shortest path the node requires more energy consumption which results in packet loss.

In the proposed work to avoid this problem, the Huffman coding packet balancer algorithm is introduced, which will compress the data without any packet loss as well as less energy consumption. Based on the energy consumption the residual energy will increase which will help in getting the shortest path along the source to destination. The throughput, by which they receive the packets time to time increases. This shows that the delay is reduced when the data is transferred from source to destination. The security level is increased with the computation cost which will avoid the routing cost.

The packet balancer will compare the packet size with node QOS and will allow the packet to find the shortest path. The node which is very near will be detected and this results in the reduction of time delay. This will allow the data to transfer with the minimum time delay as well as energy consumption. Sparse recovery is used to reconstruct the path when there is high density of node. When the source is far away from the destination then it will not have a direct path so it will a shortest path with the QOS nodes. This is done by sparse recovery to manage the data transfer speed and reduce the time delay. The main advantage to choose our system is to minimize the loss of packets during transfer and reduce the time delay with additional parameters.

\section{Related Works}

Keeping the power as major factor they introduced the LEACH protocol [1] by which the consumption of power is reduced. The energy depleted sensor can no longer work properly until it is replenished. Mainly focusing on this issue to overcome the power 
consumption which will lead to the drain in energy. It is a self organizing protocol with multi hop hierarchical protocol.

Multiple overlapping wireless sensor networks are placed in the same area, their power and energy should be made adequate with the cooperative forwarding technique is introduced [2]. The sensors which are deployed closely should transmit the data without any collisions. This introduced the heterogeneity in the network.

The grid topology is introduced through which the data can be collected in multiple directions [3] using the clustering networks. Based on the distance each node will adjust transmission power. This grid topology mainly reduces the distance between the nodes as they are located close to each other in specified paths. The data is collected with mules which are mobile entities that will collect the data from leftmost to the rightmost.

To overcome the compressive data gathering problem in collecting the data in large scales, a decentralized method is introduced [4] [15] so that the data can be collected easily and can be compressed. The delay before the data transfer gets started known latency problem is minimized. Scheduling problem is reduced with the interference localization and latency is reduced with in it. The distributed method showed the energy efficiency in the compressed data while gathering. Mainly focused on energy efficiency and latency during the gathering of data from the sensor nodes.

Sparse data collection with the compressive sensing is done [5] with new algorithm MAPDA which will reduce the sparse rate by detecting it. In compressed sensing the data will be sparse in some conditions and using this new algorithm it can be detected. The data represented in matrix and when any sensor node value is represented with zero or null then the value will be detected and adding a random value the node will transfer the data packet to the destination finding a path. This algorithm will save energy compared to the client server algorithm used in previous.

With the previous work the data collection as a main problem the single hop technique [6] where the nodes transferred the data to sink node. By developing the multi hop way to overcome this issue with the time efficient way and data loss.

\section{Implementation of Data Compression using Huffmann Coding Packet Balancer}

Our proposed work is to minimise the energy consumption and packet loss while making the data to compress. To reduce the time delay and the collision of data due to any intermediate node transfer.

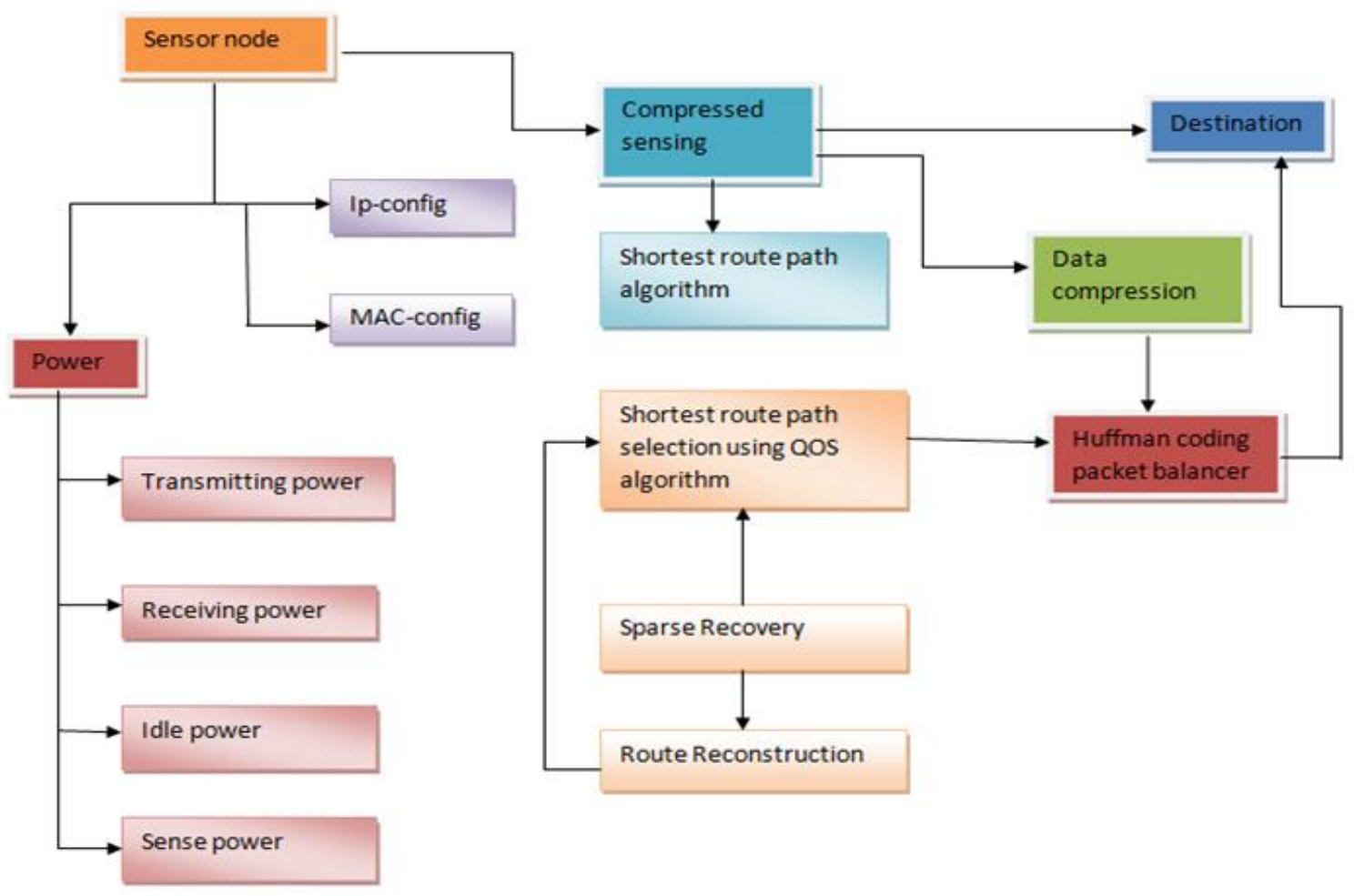

Fig. 1: Architecture diagram of data compression in wireless sensor network

In fig[1] the sensor will collect the data with the configured IP and MAC addresses. The various powers will include to transfer the data packet from source to the destination without any intermediate distractions. To make the data transfer easy the compressed sensing takes place which involves in compressing the data. The shortest path algorithm is used to obtain a shortest path with less number of nodes interacting between the source and the destination nodes.

The Huffman coding packet balancer sets the best intermediate node by comparing the nodes QOS based on the transmitting power, receiving power ideal power, sensing power. On comparing with QOS of node the best intermediate node path is selected to send compressed packet from source to the destination. Due to this the packet loss and energy consumption are mainly reduced.

The shortest path will be detected using the packet balancer which helps the packets from not losing the quality or time delay.
The source node which is in depth from the destination node will require a shortest path with the least sparse rate. To detect and protect the packet from collision due to energy loss the sparse recovery technique is used.

The Huffman coding is mainly responsible for compressing the data in very efficient way so that there should not be any data packet loss during the source to destination transfer. The packet balancer will compare the packet size with the nearest node to get the shortest distance with minimum nodes to reach the destination without time delay and energy efficient.

In wireless sensor networks the data compression plays a major role due to which the data transfer is occurring in time. But due to some parameters the data collision is getting occurred which will result in time delay and energy consumption. To overcome all these parameters. we have proposed our Huffman coding system with which the data can be compressed without any data collisions and loss of data. In addition with the packet balancer which will allow the 
node to find the shortest path with the better neighboring node, good quality and charge. Using the sparse recoverywe can find the shortest path when there is high density of nodes.

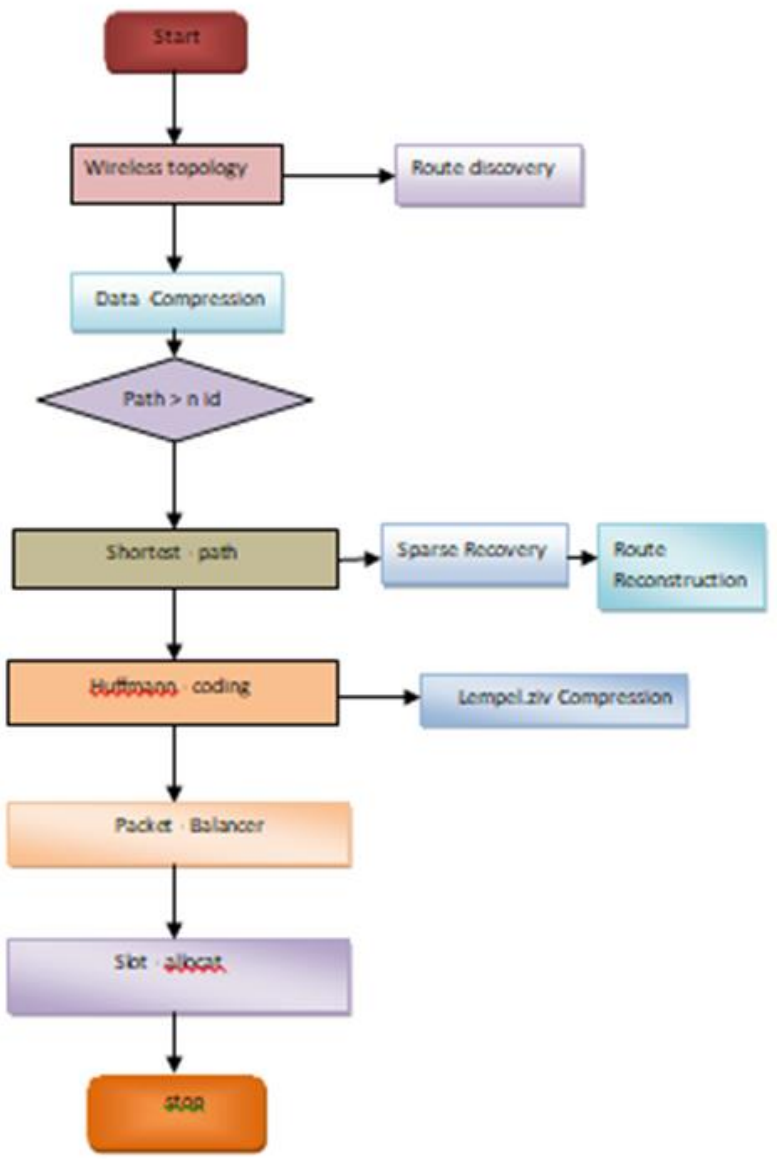

Fig. 2: Process of data compression using Huffman-coding algorithm

In fig [2] the process of data compression with Huffman coding is shown in a step by step diagram. Between the source to destination the whole data transfer takes place with the compressed data. With a wireless grid topology we are dividing the network into grids. The shortest path will be determined and with the help of sparse recovery the route reconstruction takes place if there is any intermediate node delay. Using the Huffman coding algorithm the compression of data takes place without any packet loss or energy wastage.

The packet balancer then will create a shortest path with the best neighboring nodes with its QOS which will help not to lose its quality and reduces time delay. Then after finding the shortest path finally the slot allocation will be done. The Huffman coding algorithm is mainly responsible in compressing the data without any intermediate loss or occurrence of any error during transfer. The packet balancer will allow the node to connect to the node which is in shortest distance with the

\section{Algorithm}

\section{Step wise algorithm}

Step: 1 create a grid topology with more number of nodes.

Step:2 Supply a power parameters such as transmission, reception, ideal, initial energy.

Step:3 Source generates the packets and compress the message

by using Huffman coding technique.
Step:4 Route discovery process will be processed based upon the residual energy and distance calculation.

Step:5 Source analyse the multiple path based upon the QOS of the node.

Step:6 Then itoptimize the path based upon the shortest distance.

Step:7 Source have a set of fitness based neighbours and update the value in the routing table.

Step:8 During high density in the network, The sparse recovery algorithm should select the alternate path between the communication in the updated routing table.

\section{Huffman-coding}

Input:

Data_pkts, Tx_power, Rx-power,Initial_energy.

Output:

Slot_time, Delivered_pkts, rt_pkts.

1: for the marked $i d\left(1_{-} j_{-} N i d\right)$ in each query do

2: if $s r c$ can broadcast the packet with each other than

3: $\quad$ BS uses route discovery to assign route.

else

BS sends the ack_pkt to $s j$ closest to it.

ifnbr nodes of $s r c$ are the marked sensor nodes then

The admin node will optimize to the closest neighbour node

8: $\quad \operatorname{src}\left(1_{-} i_{-}\right.$Nid $)$to $s j$.

9: $\quad$ else

10: $\quad$ Srcchooses the closest and nearby sensor node $s i\left(1_{-} i_{-}\right.$ $N$ )as candidate node.

11: if $s j$ can communicate with at least one neighbour node of sithen

12: srccompress the data using Huffman coding $\mathrm{H}(1), \mathrm{H}(2), \mathrm{H}(3) . . \mathrm{H}(\mathrm{n})$

13: $\quad s r c s e a r c h e s$ the nearby nodes in other sensor group, which aren't

14: Optimize the neighbour node closest to $B S$ as next hop

15: src. Srcsearches its neighbour nodes among the other remaining sensor to overcome the sparse recovery nodes by using distance calculation

$16: F 1\left(A_{3} \mid B\right)=F 2\left(B \mid A_{3}\right) F 1\left(A_{3}\right) / F 2(B)$

$17 \quad:$ F->fitness value

18: $\quad$ end if

19: whilesrccan't communicate the node without range

20: $\quad$ ifsican communicate with at least a sparse node of nidthen 21 :break

22: else

23: $\quad$ Choose the alternate path by using distance calculation 24: end if

\section{Implementation}

\section{Output in NS2 using grid topology}




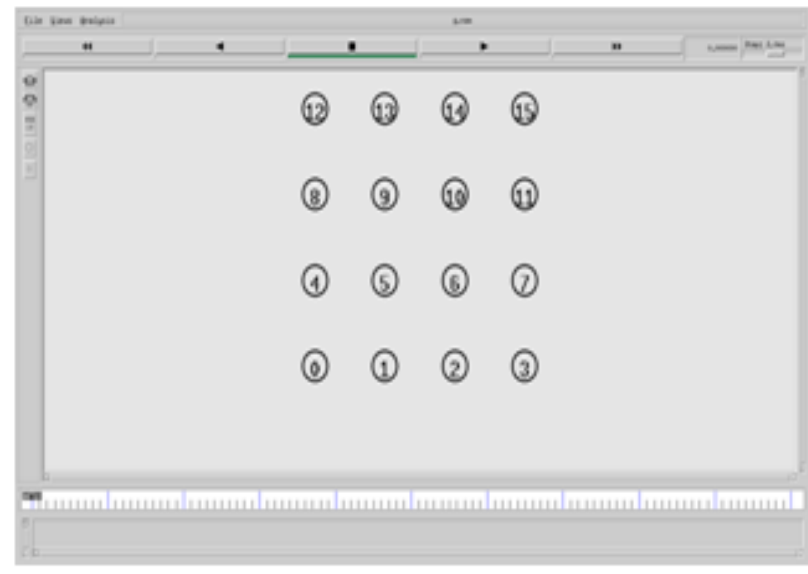

Fig. 3(a): Nodes in grid topology

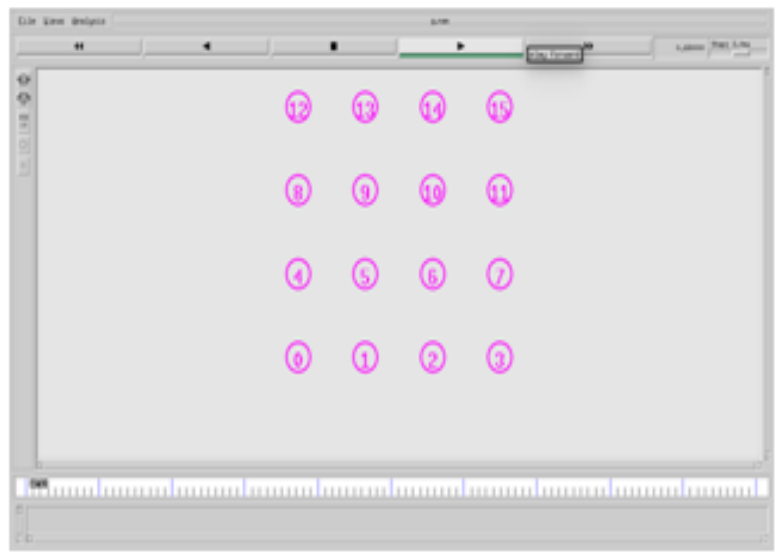

Fig. 3(b): simulation in grid topology

A wireless sensor system is a gathering of sensor nodes controlled in a helpful system. In WSN, the sensors node screens physical and ecological conditions at various areas, for example, temperature, weight, dampness, vibration and so forth. In grid topology the nodes are placed in matrx form at particular distance with equal gaps. So that the source node can get a shortest path based on the energy and QOS of the neighboring node. With the sparse recovery it will get the shortest path with minimum transaction time and time delay.

The node to node data transfer takes place in the shortest path with minimum number of intermediate nodes. To defeat this issue the sensor nodes are gathered in a type of network topology. Lattice topology in WSN has been actualized to separate the system field into networks. In this topology, the sensor nodes containing system region is slated into the nonconsolidating square network with the same size.Huffman coding packet balancer select the best path between the intermediate node are compared based on transmitting power, receiving and sensing power these measure the QOS in wireless sensor network.Lattice steering convention proposed to transmit bundles quick, use and broaden the vitality proficiency of sensor nodes to maintain a strategic distance and handle the organized movement in the system. The node in circular path will check all the near by nodes to get the best QOS node.

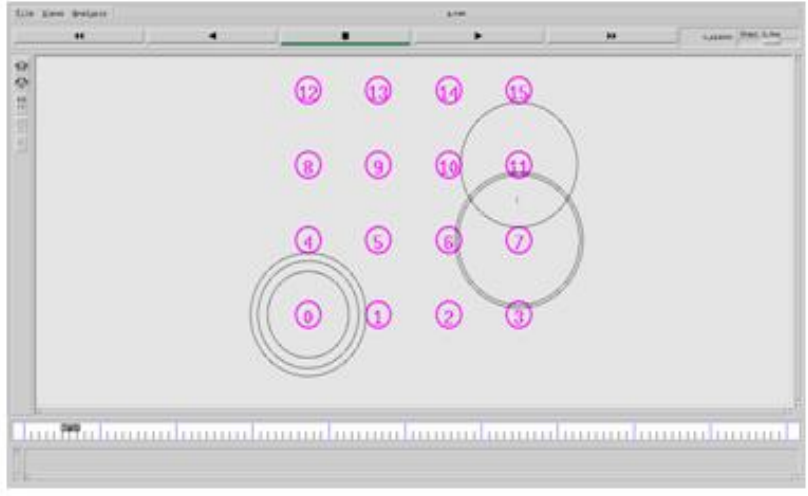

Fig. 3(c): checking QOS of nodes

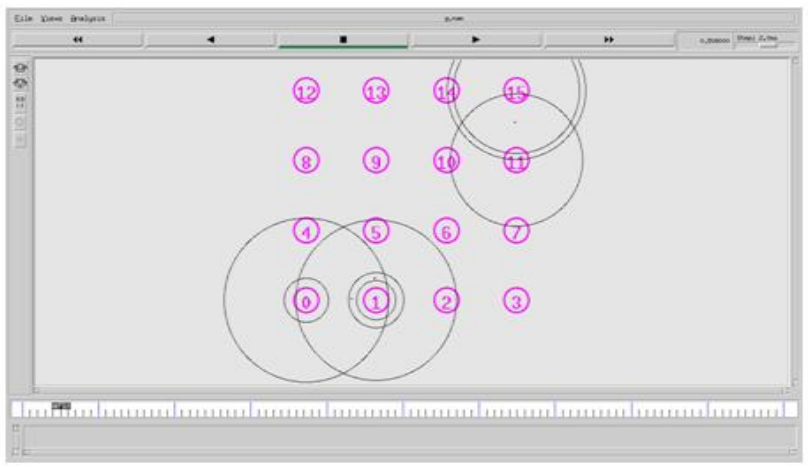

Fig. 3(d): Finding the shortest path between nodes

\section{Graph output}

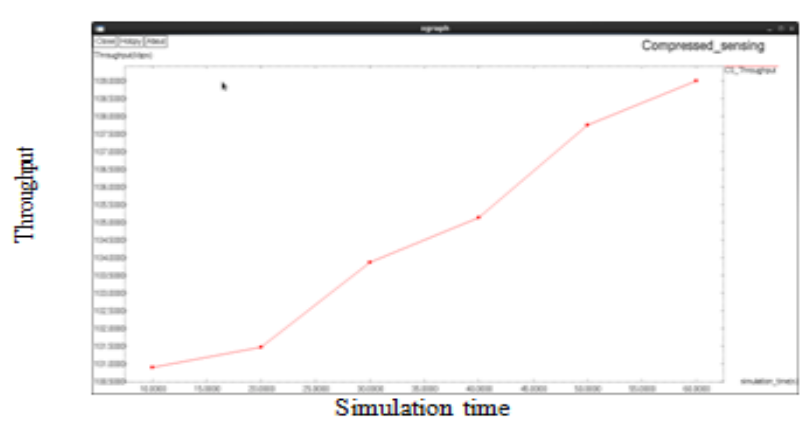

Fig. 4: Simulation time vs Throughput

Fig[4] shows the receival of packets increases time to time, with the throughput .The increase of throughput with the increase in simulation time is shown.

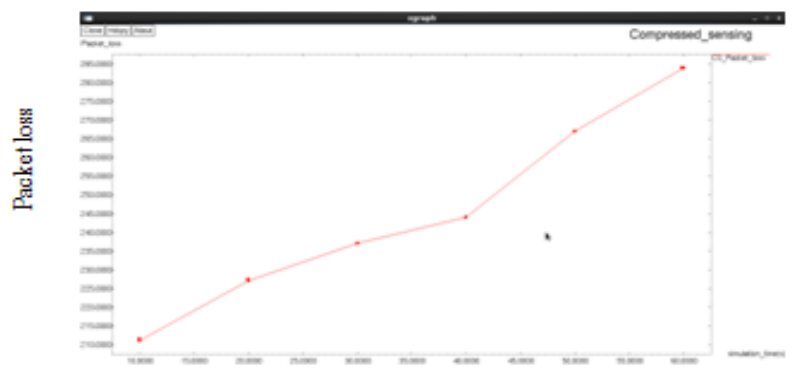

Simulation tim

Fig. 5: Simulation time vs Packet loss 
Fig[5] represents the packet loss when the simulation is done with respect to the time given. The packet loss decreases when compared to the existing system.

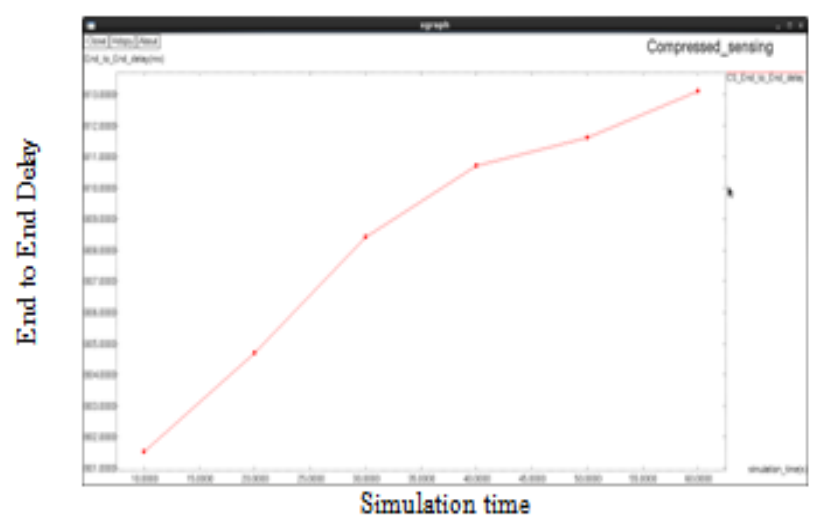

Fig. 6: Simulation time vs End to End Delay

Fig[6] represents the maximum time taken to travel from source to destination. With respect to the simulation time the end to end delay is shown which will decrease when compared to the previous system.

\section{Conclusion}

we mainly reduce the packet loss and energy consumption with the grid topology in wireless sensor networks. The Huffman coding packet balancer select the best intermediate node by comparing the nodes QOS based on the transmitting power, receiving power, ideal power, sensing power. On comparing with QOS of node the best intermediate node path is selected to send compressed packet from source to destination. Sparse recovery is used to reconstruct the path selection when there is high density of node.

\section{Acknowledgement}

It is a great honor to express our profound and sincere gratitude to our Computer Science Engineering department, SRM Institute of science and technology, Kattankulathur for encouraging and giving us support and encouragement by providing good environment to carry out this research work and to gain valuable experiences.

\section{References}

[1] Maya M. Warriera*, Ajay Kumarb, '’An energy efficient approach for routing in wireless sensor Networks" Kalady, Ernakulam, 683574, Kerala, India, 2016.

[2] Kazuhiko Kinoshita, Member, IEEE NatsukiInoue, Nonmember Yosuke Tanigawa, Member, IEEE,HidekiTode, Member, IEEE, and Takashi Watanabe, Fellow, IEEE, 'Fair Routing for Overlapped Cooperative Heterogeneous Wireless Sensor Networks"'; IEEE SENSORS JOURNAL, VOL. 14, NO. 1, JANUARY 2016.

[3] Sunil Kumar Singh, Prabhat Kumar and Jyoti Prakash Singh, "'An Energy Efficient Odd-Even Round Number Based Data Collection using Mules in WSNs'; IEEE WiSPNET 2016 conference.

[4] Dariush Ebrahimi and Chadi Assi, Senior Member, IEEE, 'On the Interaction Between Scheduling and Compressive Data Gathering in Wireless Sensor Networks"; IEEE TRANSACTIONS ON WIRELESS COMMUNICATIONS, VOL. 15, NO. 4, APRIL 2016.

[5] Qiang Wang Member IEEE, CuicuiLv, Yi Shen Member IEEE, Jinming Chen, 'Compressed Sensing and Mobile Agent Based Sparse Data Collection in Wireless Sensor Networks" IEEE 2015.
[6] W. U.Bajwa, J. D. Haupt, A. M. Sayeed, and R. D. Nowak, "Joint source-channel communication for distributed estimation in sensor networks, 'IEEE Transactions on Information Theory, vol.53, no.10, pp.3629-3653, 2007

[7] J. Yick, B. Mukherjee, and D. Ghosal, "Wireless sensor network survey," Computer networks, vol.52, no.12, pp.2292-2330,2008.

[8] M. Chen, S. Gonzalez, and V. C. Leung,"Applications and design issues for mobile agents in wireless sensor networks," IEEE Wireless Communications,vol.14, no.6, pp.20-26,2007.

[9] R.Baraniuk, “Compressive sensing,"IEEE signal processing magazine, vol.24, no.4. pp.118-120, 2007.

[10] D.L. Donoho, “Compressed sensing,"IEEETransactions on Information Theory,vol.52, no.4,pp.1289-1306, 2006.

[11] E. J. Cands, M. B. Wakin,"An introduction to compressive sampling,'IEEE Signal Processing Magazine, vol.25, no.2, pp.2130,2008 .

[12] W. U.Bajwa, J. D. Haupt, A. M. Sayeed, and R. D. Nowak, "Joint source-channel communication for distributed estimation in sensor networks,"IEEE Transactions on Information Theory, vol.53, no.10, pp.3629-3653, 2007.

[13] S. Ji, R. Beyah, and Z. Cai, "Snapshot and continuous data collection in probabilistic wireless sensor networks," IEEE Trans. Mobile Comput., vol. 13, no. 3, pp. 626-637, Mar. 2014.

[14] Srbinovski, B., Magno, M., O'Flynn, B., Pakrashi, V., \&Popovici, E.Energy aware adaptive sampling algorithm for energy harvesting wireless sensor networks. Sensors Applications Symposium (SAS), IEEE 2015; 1-6.

[15] Amarlingam M, Pradeep Kumar Mishra, K. V. V. Durga Prasad, P Rajalakshmi,"Compressed Sensing for Different Sensors: A Real Scenario for WSN and IoT " in Proc. Indian Institute of Technology Hyderabad, 2016

[16] T. Padmapriya, V.Saminadan, "Performance Improvement in long term Evolution-advanced network using multiple imput multiple output technique", Journal of Advanced Research in Dynamical and Control Systems, Vol. 9, Sp-6, pp: 990-1010, 2017.

[17] M. Rajesh, Manikanthan, "GET-UP-AND-GO EFFICIENT MEMETIC ALGORITHM BASED AMALGAM ROUTING PROTOCOL", International Journal of Pure and Applied Mathematics, ISSN NO:1314-3395, Vol-116, No. 21, Oct 2017. 